\title{
Correlation Between mpMRI Staging and Final Surgical Pathology in Prostate Cancer
}

\author{
Alireza Lashay ${ }^{1}$, Jafar Gholivandan ${ }^{1}$, Yaghoob Sehri ${ }^{1}$, Amirreza Elahian² \& Mahyar Ghafari ${ }^{3}$ \\ ${ }^{1}$ Department of Urology, shahid modarres Hospital, Shahid Beheshti University of medical sciences, Tehran, \\ Iran \\ ${ }^{2}$ Department of Urology, Khatam-ol-anbia Hospital, Tehran, Iran \\ ${ }^{3}$ Department of Radiology, Shahid Beheshti University of Medical Sciences, Tehran, Iran \\ Correspondence: Jafar Gholivandan, Department of Urology, shahid modarres Hospital, Shahid Beheshti \\ University of medical sciences, Tehran, Iran. E-mail: gholivandani@gmail.com
}

Received: October 30, 2019

Accepted: January 2, 2020

Online Published: March 30, 2020

doi:10.5539/jmbr.v10n1p6

URL: https://doi.org/10.5539/jmbr.v10n1p6

\begin{abstract}
Purpose: We evaluated the role of multiparametric magnetic resonance imaging (mpMRI) in the diagnosis of prostate cancer and predicting of surgical staging of prostate cancer.

Materials and Methods: The study was done in 110 subjects who got mpMRI before radical prostatectomy in our hospital from 2016 to 2019. Preoperative mpMRI findings of 110 were compared to surgical pathology results following radical Prostatectomy. A comparison was made between pathologic staging of prostate cancer and the mpMRI findings.

Results: pathologic evaluation confirmed prostate cancer foci (237) were recognized in 110 subjects. Generally, mpMRI sensitivity of $46.4 \%$ was found for prostate cancer detection (110/237). Pathological tumor volume was a significant predictor of prostate cancer detection using mpMRI. In $33 \%$ of the cases, the pathologic staging is precisely similar to mpMRI and in $43 \%$ of the cases, there was a slight difference between the pathologic staging and staging by mpMRI but the cancer was confined to the prostate.in $24 \%$ of the cases, there was a significant difference between the pathologic staging and staging by mpMRI. The mpMRI was not able to identify the significant cancer in $24 \%$ of the cases.

Conclusion: The preoperative mpMRI was useful in detecting prostate cancer and in predicting surgical staging. However, the detection of $24 \%$ of clinically significant cancer was missed using mpMRI. As we move toward personalized medicine, use of MRI to biopsy each man's prostate differently rather than based on a pre-defined 12 core seems to be supported in the recent literature.
\end{abstract}

Keywords: Multiparametric MRI (mpMRI), Prostate Cancer (PCa), Radical Prostatectomy (RP)

\section{Introduction}

Malignant neoplasms have remained as a leading cause of death worldwide (Abdel-Sattar et al., 2018). Cancer is the uncontrolled proliferation of abnormal cells that leads to a malignant growth, and ultimately severe morbidity and mortality (Alshammari, 2018). The diagnostic pathway for prostate cancer detection is initiated on prostate-specific antigen (PSA) level and digital rectal exam (DRE). Use of PSA as a screening tool followed by systematic transrectal ultrasound-guided (TRUS) biopsy has resulted in increased detection of prostate cancer with stage migration toward low-risk disease (Mottet et al., 2017). For evaluation of the clinical staging, routine diagnostics (i.e., digital rectal examination, serum prostate-specifc antigen (PSA) level, transrectal ultrasound, and Gleason score) are insufficient (Carroll et al., 2016). MRI findings in patients are of importance in detection and management of disorders (Mosarrezaii et al., 2017). Multiparametric MRI has become a valuable tool in the diagnosis of prostate cancer. Although most large, high grade cancers are visible on mpMRI, intermediate grade and low volume cancers are often difficult to identify. Furthermore, the usefulness of MRI for determining the true size and shape of a tumor remains incompletely characterized. (Ukimura et al., 2013)

Multiparametric MRI is increasingly used in prostate cancer assessment for its diagnosis and detection and for staging and risk stratification (Thompson, Lawrentschuk, Frydenberg, Thompson, \& Stricker, 2013; Felker, Margolis, Nassiri, \& Marks, 2016). A concern regarding mpMRI is the considerable interobserver variability 
(Fedorov, Vangel, Tempany, \& Fennessy, 2017; Marin et al., 2017). Mp-MRI has been used to assess prostate cancer aggressiveness and to identify anteriorly located tumors before and during active surveillance. With the technological advancement of imaging modalities used in prostate cancer assessment, Introduction of the Prostrate Imaging Reporting and Data System (PI-RADS), constitutes a globally accepted standard for the detection, scoring and reporting of suspicious lesions on mpMRI (Weinreb et al., 2016). In two studies, improved detection of clinically significant cancer and a decrease in the identification of indolent cancer were demonstrated with the use of mpMRI in conjunction with PI-RADS and a subsequent targeted biopsy (Borkowetz et al., 2016; Toner et al., 2017). However, as mpMRI with PI-RADS is rapidly being adopted for prostate cancer detection and surveillance, additional efforts to identify the diagnostic accuracy of mpMRI in prostate cancer detection and its ability to predict tumor aggressiveness are warranted to determine if it should play a decisive role in prostate cancer management.

Thus, the essential aims of present study were to evaluate the diagnostic accuracy of mpMRI in the detection of prostate cancer and prediction of pathological staging, and to directly compare the findings on mpMRI with the histological findings from the radical prostatectomy specimen.

\section{Materials and Methods}

For this study, we have identified a hundred and ten patients who had experienced mpMRI prior to radical prostatectomy for localized prostate cancer between 2016 and 2019 at our institute. Inclusion criteria were histologically confirmed prostate adenocarcinoma. Finally, 110 patients were selected for inclusion in the study. Data were collected in a database in which patient demographics, clinical results, prostate biopsy results, mpMRI-related information and final histopathological results were documented.

A total of 110 patients underwent mpMRI at the Department of Radiology of our Hospital. In the Department of Radiology, all mpMRI of the prostate is performed on a 3-Tesla MRI system (Siemens Medical Solutions Germany). The mpMRI protocol of the prostate included T2-weighted images in transverse and coronal orientation, T1-weighted images in transverse orientation, diffusion weighted images in transverse orientation, dynamic contrast enhanced imaging in transverse orientation and contrast enhanced T1-weighted images with fat suppression in transverse orientation. The total MRI acquisition time was 30min. Endorectal coil was not used. The mpMRI findings have been scored by using PI-RADS version 2 .

The prostate specimens were reviewed by a dedicated uropathologist who was blinded to the mpMRI findings. All prostatic biopsies and radical prostatectomy specimens were investigated at the Pathology unit of our Hospital. The whole prostate was prepared in $3-5 \mathrm{~mm}$ increments and embedded in paraffin. These sections were cut and stained with haematoxylin and eosin for microscopic examination. Each individual tumor focus in the radical prostatectomy specimen graded according to the Gleason grading system. The highest Gleason score recorded per tumor foci was equated to a corresponding score using the new grading system For example, grade group 1 equated to a Gleason score of $3+3$ (the least aggressive), grade group 2 was the equivalent of a Gleason score of $3+4$, grade group 3 amounted to a Gleason score of $4+3$, grade group 4 equated to a Gleason score of $4+4,3+5$ and $5+3$ and a grade group 5 was the equivalent of a Gleason score of 9-10 (the most aggressive).The pathological index tumor was defined as the tumor in the highest-grade group. Based on the calculation of tumor volume, clinically significant cancer was defined as a tumor in grade group 2 with a cancer volume of $0.5 \mathrm{cc}$. (Weinreb et al., 2016; Wolters et al., 2011; Lee, Ku, Park, Lee, \& Ha, 2018).

Data were analysed using SPSS v.23.0. Categorical data are presented as absolute and relative frequencies. Continuous variables are described using mean values, complemented by median and range values. Linear-by-linear association and the chi-square test were used to compare the rate of tumors detected and missed on mpMRI, according to the pathological features. Spear- man's rank-correlation coefficient was used to determine an association between the PI-RADS score and the pathological features of grade and volume. Cut-off values for pathological tumor volume detected by mpMRI were obtained using the Youden index. A P value $<0.05$ was taken to indicate statistical significance.

\section{Results}

The demographic characteristics of the study are shown in Table 1. The median age of the 110 men who underwent mpMRI prior to RP was 63 years (50-75 years) and median PSA was $10.7 \mathrm{ng} / \mathrm{mL}$ (5.1-30). The median time taken from performing mpMRI to conducting RP was 29 days (7-63 days). Unique pathologically confirmed prostate cancer foci (237) were identified in 110 patients, 39 (36\%) of whom had solitary and 68 (64\%) of whom had multifocal tumors. mpMRI successfully identified 249 lesions.

Overall sensitivity of $46.4 \%$ was achieved using mpMRI with PI-RADS for the detection of prostate cancer 
(110/237). In total, $106(44.7 \%)$ tumor lesions on radical prostatectomy specimen were identified as clinically significant cancer. The sensitivity, specificity, negative predictive value (NPV), PPV and accuracy of detection of clinically significant cancer were $75.5 \%, 77.0 \%, 79.8 \%, 72.7 \%$ and $76.3 \%$, respectively. A total of $75.7 \%$ $(81 / 107)$ of the pathological index tumors in the RP specimens were detected using mpMRI with PI-RADS. The median pathological index tumor volume was $2.31 \mathrm{cc}(0.10-11.21 \mathrm{cc})$. The sensitivity, specificity, NV, PPV, and accuracy of detection of pathological index tumors were $75.7 \%, 77.7 \%, 79.5 \%, 73.6 \%$ and $76.8 \%$.

Table 1. The demographic characteristics for 110 men

\begin{tabular}{|c|c|}
\hline Age, years; median (min; max) & $63(50 ; 75)$ \\
\hline PSA, ng/mL; median (min; max) & $10.7(5.1 ; 30)$ \\
\hline Positive findings in DRE & 37 \\
\hline Prostate volume, $\mathrm{mL} ;$ median $(\min ; \max )$ & $41(15 ; 112)$ \\
\hline Time from biopsy to RP, days; median (min; max) & $29(7 ; 63)$ \\
\hline \multicolumn{2}{|l|}{ Histological findings } \\
\hline \multicolumn{2}{|l|}{ Tumor multifocality } \\
\hline Solitary & $39(36.4)$ \\
\hline Multifocal & $68(63.6)$ \\
\hline \multicolumn{2}{|l|}{ Number of multifocal tumor } \\
\hline 2 foci & $29(27.1)$ \\
\hline 3 foci & $21(19.6)$ \\
\hline 4 foci & $14(13.1)$ \\
\hline$\geq 5$ foci & $4(3.7)$ \\
\hline \multicolumn{2}{|l|}{ Pathological stage } \\
\hline pT2a & $21(19.6)$ \\
\hline $\mathrm{pT} 2 \mathrm{~b}$ & $22(20.6)$ \\
\hline pT2c & $52(48.6)$ \\
\hline pT3a & $3(2.8)$ \\
\hline pT3b & $9(8.4)$ \\
\hline \multicolumn{2}{|l|}{ Grade group } \\
\hline Grade group 1 (Gleason score $3+3$ ) & $15(14.0)$ \\
\hline Grade group 2 (Gleason score $3+4$ ) & $44(41.1)$ \\
\hline Grade group 3 (Gleason score 4+3) & $22(20.6)$ \\
\hline Grade group 4 (Gleason score 8$)$ & $7(6.5)$ \\
\hline Grade group 5 (Gleason score 9-10) & $19(17.8)$ \\
\hline \multicolumn{2}{|l|}{ mpMRI findings } \\
\hline \multicolumn{2}{|l|}{ PIRADS score of each tumor focus } \\
\hline PIRADS $\leq 2$ & $127(51.0)$ \\
\hline PIRADS 3 & $31(12.4)$ \\
\hline PIRADS 4 & $50(20.1)$ \\
\hline PIRADS 5 & $41(16.5)$ \\
\hline
\end{tabular}

In addition, higher PI-RADS scores were significantly associated with increased tumor volume $(\mathrm{P}<0.001)$. Pathological index tumor volume was the strongest predictor of tumor detection by mpMRI with PI-RADS $(\mathrm{P}=$ 0.03 ). In $33 \%$ of the cases, the pathologic staging is precisely similar to mpMRI and in $43 \%$ of the cases, there was a slight difference between the pathologic staging and staging by mpMRI but the cancer was confined to the prostate.in $24 \%$ of the cases, there was a significant difference between the pathologic staging and staging by mpMRI. The mpMRI was not able to identify the significant cancer in $24 \%$ of the cases.

\section{Discussion}

The aim of our study was to determine the accuracy of MRI for predicting pathological staging of prostate cancer. The main role of mpMRI with PI-RADS in prostate cancer diagnosis is to identify clinically significant cancer. Although there is no general consensus on the definition of clinically significant $\mathrm{PCa}$, clinically significant cancer is defined on pathology as grade group 2 with a cancer volume of $0.5 \mathrm{cc}$ (Weinreb et al., 2016; Wolters et al., 2011). In present study, 3T mpMRI with PI-RADS demonstrated overall sensitivity of $46.4 \%$ and specificity of $75.5 \%$ in detecting clinically significant cancer. These results are consistent with those of previous studies. Jesse et al. reported overall sensitivity and specificity of $47 \%$ and $72 \%$, respectively, for the detection of 
clinically significant cancer,12 and Bratan et al. reported 53-59\% overall sensitivity (Bratan et al., 2013). Although the individual sequences are useful, T2WI in combination with two functional sequences has been shown to provide better characterization of tumor in the prostate ([42, 43, and 44). In a diagnostic meta-analysis of seven studies, de Rooij et al. revealed a high overall sensitivity and specificity on accuracy of mp-MRI using T2WI, DWI and DCE MRI. Pooled sensitivity and specificity were 0.74 and 0.88 , respectively, with negative predictive value (NPV) ranging from 0.65 to 0.94 (de Rooij, Hamoen, Fütterer, Barentsz, \& Rovers, 2014).

Although, theoretically, mpMRI with PI-RADS is known to be able to detect intermediate- to high-grade cancers with volumes $0.5 \mathrm{cc}$ (Weinreb et al., 2016). Our data showed that $24 \%$ of clinically significant cancer and pathological index tumors were missed using this approach.

Wang et al. reported that a decrease in the degree of intensity in the peripheral zone on T2WI correlated with Gleason grade (Wang, Mazaheri, Zhang, Ishill, Kuroiwa, \& Hricak, 2008). Similarly, in the case of DWI, the diffusion of water molecules was more restricted in tightly packed high-grade prostate cancer compared to that in low-grade $\mathrm{PCa}$, which is more loosely packed or more normal prostate-like tissue architecture, thus depicting hyperintense focal lesions on high b-value DWI and hypointense focal lesions on ADC mapping. Elsewhere, an inverse correlation between quantitative ADC mapping values and Gleason grade was demonstrated (Hambrock et al., 2011; Nowak et al., 2016). Although our data and those in previous studies suggest that mpMRI with PI-RADS can be used as a tool to predict prostate cancer aggressiveness, attention should be paid to the fact that $36 \%$ of lesions classified as PI-RADS 2 were grade group 2. Borkowetz et al. and Truong et al. reported that most tumors that could not be visualized on mpMRI were found to be cribriform ones, which are currently interpreted as Gleason pattern 4 (Borkowetz et al., 2016; Truong, Hollenberg, Weinberg, Messing, Miyamoto, \& Frye, 2017). It was suggested that the more open cellular architecture of cribriform tumors, compared to Gleason pattern 4 or 5 tumors, could lead to misinterpretation of high-grade tumors as normal architecture or low-grade tumors on mpMRI.

The value of using staging and treatment planning for prostate cancer has been demonstrated in contemporary studies on mpMRI in prostate cancer (McClure et al., 2012; Somford et al., 2013). In addition, it was shown in a recent prospective trial that when used with targeted biopsy, mpMRI improved the detection of significant prostate cancer and reduced the need for unnecessary invasive tests (Ahmed et al., 2017).

\section{Conclusion}

We evaluated the ability mpMRI with PI-RADS (version 2) to detect prostate cancer and a direct comparison was made with the pathology findings for RP specimens. Sensitivity of $76 \%$ was found for both clinically significant cancer detection and the findings for pathological index tumors. A moderate and significant correlation was observed between a high PI-RADS score and a high pathological grade, tumor volume, index tumor status and clinically significant cancer status. Pathological index tumor volume was the strongest predictor of tumor detection. The mpMRI was not able to identify the significant cancer in $24 \%$ of the cases. MRI/US-fusion biopsy was associated with a higher detection rate of clinically significant prostate cancer while taking fewer cores, especially in patients with prior negative biopsy. As we move toward personalized medicine, use of MRI to biopsy each man's prostate differently rather than based on a pre-defined 12 core seems to be supported in the recent literature.

\section{Conflict of interests}

The authors declare that there is no conflict of interests regarding the publication of this paper.

\section{References}

Ahmed et al. (2017). Diagnostic accuracy of multi-parametric MRI and TRUS biopsy in prostate cancer (PROMIS): A paired validating confirmatory study. Lancet, 389, 815-822.

Borkowetz et al. (2016). Direct comparison of multiparametric magnetic resonance imaging (MRI) results with final histopathology in patients with proven prostate cancer in MRI/ultrasonography-fusion biopsy. BJU Int, $118,213-220$.

Bratan et al. (2013). Influence of imaging and histological factors on prostate cancer detection and localisation on multiparametric MRI: A prospective study. Eur Radiol, 23, 2019-2029.

Carroll et al. (2016). NCCN guidelines insights: Prostate cancer early detection, version 2.2016. J Natl Compr Canc Netw, 14, 509-519.

de Rooij, M., Hamoen, E. H., Fütterer, J. J., Barentsz, J. O., \& Rovers, M. M. (2014). Accuracy of multiparametric MRI for prostate cancer detection: A meta-analysis. AJR Am J Roentgenol, 202, 343-51. 
Fedorov, A., Vangel, M. G., Tempany, C. M., \& Fennessy, F. M. (2017). Multiparametric magnetic resonance imaging of the prostate: repeatability of volume and apparent diffusion coefficient quantification. Invest Radiol, 52, 538-546.

Felker, E. R., Margolis, D. J., Nassiri, N., \& Marks, L. S. (2016). Prostate cancer risk stratification with magnetic resonance imaging. Urol Oncol, 34, 311-319.

Futterer et al. (2015). Can clinically significant prostate cancer be detected? With multiparametric magnetic resonance imaging? A systematic review of the literature. Eur Urol, 68, 1045-1053.

Abdel-Latif Abdel-Sattar, S., Abdel-Fatah Ibrahim, H., Abd Elwahab El Sayed, H. (2018). Knowledge, Attitude and Practices of Working Women in Tabuk University Regarding Breast Cancer. International Journal of Pharmaceutical Research \& Allied Sciences, 7(3),198-208

Hambrock et al. (2011). Relationship between apparent diffusion coefficients at 3.0-T MR imaging and Gleason grade in peripheral zone prostate cancer. Radiology, 259, 453-461.

Jung et al. (2018). Multiparametric MR imaging of peripheral zone prostate cancer: Effect of postbiopsy hemorrhage on cancer detection according to Gleason score and tumour volume. Br J Radiol, 20180001.

Katelaris, N. C., Bolton, D. M., Weerakoon, M., Toner, L., Katelaris, P. M., \& Lawrentschuk, N. (2015). Current role of multiparametric magnetic resonance imaging in the management of prostate cancer. Korean J Urol, $56,337-345$.

Mosarrezaii, A., Hedayati Asl, A., Ghafouri, A. A. (2017). Assessment of Prevalence of MRI Findings in Epileptic Patients of Imam Khomeini Hospital, Urmia, Iran during 2010-2011. Journal of Advanced Pharmacy Education \& Research, 7(4), 473-478.

Le et al. (2015). Multifocality and prostate cancer detection by multiparametric magnetic resonance imaging: Correlation with whole-mount histopathology. Eur Urol, 67, 569-576.

Lee, C. H., Ku, J. K., Park, W. Y., Lee, N. K., \& Ha, H. K. (2018). Comparison of the accuracy of multiparametric magnetic resonance imaging (mpMRI) results with the final pathology findings for radical prostatectomy specimens in the detection of prostate cancer. Asia-Pac J Clin Oncol, 1-8.

Marin et al. (2017). Comparison of semi-automated and manual methods to measure the volume of prostate cancer on magnetic resonance imaging. Diagn Interv Imaging, 98, 423-428.

McClure et al. (2012). Use of MR imaging to determine preservation of the neurovascular bundles at robotic-assisted laparoscopic prostatectomy. Radiology, 262, 874-883.

Mottet et al. (2017). EAU-ESTRO-SIOG guidelines on prostate cancer. Part 1: Screening, diagnosis, and local treatment with curative intent. Eur Urol, 71, 618-629.

Nowak et al. (2016). The value of ADC, T2 signal intensity, and a combination of both parameters to assess Gleason score and primary Gleason grades in patients with known prostate cancer. Acta Radiol, 57, 107-114.

Perera, M., Lawrentschuk, N., Bolton, D., \& Clouston, D. (2014). Comparison of contemporary methods for estimating prostate tumour volume in pathological specimens. BJU Int, 113(Suppl 2), 29-34.

Somford et al. (2013). The predictive value of endorectal 3 Tesla multiparametric magnetic resonance imaging for extraprostatic extension in patients with low, intermediate and high risk prostate cancer. $J$ Urol, 190, $1728-1734$.

Thompson, J., Lawrentschuk, N., Frydenberg, M., Thompson, L., \& Stricker, P. (2013). The role of magnetic resonance imaging in the diagnosis and management of prostate cancer. BJU Int., 112(Suppl 2), 6-20.

Toner et al. (2017). Multiparametric magnetic resonance imaging for prostate cancer-a comparative study including radical prostatectomy specimens. World J Urol, 35, 935-941.

Alshammari, F. D. (2018). Do Non-Viral Microorganisms Play a Role in the Aetiology of Human Cancers? A Review. Int.J. Pharm. Res. Allied Sci., 2018, 7(4), 179-185.

Truong, M., Hollenberg, G., Weinberg, E., Messing, E. M., Miyamoto, H., \& Frye, T. P. (2017). Impact of Gleason subtype on prostate cancer detection using multiparametric magnetic resonance imaging: Correlation with final histopathology. J Urol, 198, 316-321.

Ukimura et al. (2013). Contemporary role of systematic prostate biopsies: Indications, techniques, and implications for patient care. Eur Urol, 63, 214-230. 
Ullrich et al. (2017). Magnetic resonance imaging of the prostate at 1.5 versus 3.0T: A prospective comparison study of image quality. Eur J Radiol, 90, 192-197.

Wang, L., Mazaheri, Y., Zhang, J., Ishill, N. M., Kuroiwa, K., \& Hricak, H. (2008). Assessment of biologic aggressiveness of prostate cancer: Correlation of MRsignal intensity with Gleason grade after radical prostatectomy. Radiology, 246, 168-176.

Weinreb et al. (2016). PI-RADS prostate imaging - reporting and data system: 2015, Version 2. Eur Urol, 69, $16-40$.

Wolters et al. (2011). A critical analysis of the tumor volume threshold for clinically insignificant prostate cancer using a data set of a randomized screening trial. J Urol, 185, 121-125.

\section{Copyrights}

Copyright for this article is retained by the author(s), with first publication rights granted to the journal.

This is an open-access article distributed under the terms and conditions of the Creative Commons Attribution license (http://creativecommons.org/licenses/by/4.0/). 